HUPD-93-03

January 1993

\title{
Convenient versus unique effective action formalism in 2d dilaton-Maxwell quantum gravity
}

\author{
E. ELIZALDE円 \\ Department E.C.M., Faculty of Physics, University of Barcelona, \\ Diagonal 647, 08028 Barcelona, Spain \\ S. NAFtulin \\ Institute for Single Crystals, 60 Lenin Ave., 310141 Kharkov, Ukraine \\ and \\ S.D. Odintsov? \\ Department of Physics, Faculty of Science, Hiroshima University, \\ Higashi-Hiroshima 724, Japan
}

\begin{abstract}
The structure of one-loop divergences of two-dimensional dilaton-Maxwell quantum gravity is investigated in two formalisms: one using a convenient effective action and the other a unique effective action. The one-loop divergences (including surface divergences) of the convenient effective action are calculated in three different covariant gauges: (i) De Witt, (ii) $\Omega$-degenerate De Witt, and (iii) simplest covariant. The on-shell effective action is given by surface divergences only (finiteness of the $S$-matrix), which yet depend upon the gauge condition choice. Off-shell renormalizability is discussed and classes of renormalizable dilaton and Maxwell potentials are found which coincide in the cases of convenient and unique effective actions. A detailed comparison of both situations, i.e. convenient vs. unique effective action, is given. As an extension of the procedure, the one-loop effective action in two-dimensional dilaton-Yang-Mills gravity is calculated.
\end{abstract}

PACS: $04.50,03.70,11.17$.

\footnotetext{
${ }^{1}$ E-mail address: eli @ ebubecm1.bitnet

${ }^{2}$ On sabbatical leave from Tomsk Pedagogical Institute, 634041 Tomsk, Russia. E-mail address: odintsov@ theo.phys.sci.hiroshima-u.ac.jp
} 


\section{Introduction}

Black hole physics and early universe physics are issues in which quantum gravity effects are expected to be significant. Some years have elapsed since the earliest attempts [13] and we still do not have a consistent description of quantum black holes (specially, of their final states). One of the main reasons is that gravity must be quantized for such considerations; however, Einstenian quantum gravity is not consistent due to nonrenormalizability in four dimensions, and modifications of the same still have similar (or sometimes additional) drawbacks.

In such situation it is quite advisable to study solvable toy models where all these problems may be simplified drastically while keeping still many of the good properties of the more realistic theory. Two-dimensional (2d) quantum gravity with matter can be considered as such a toy model [4]. This theory is multiplicatively renormalizable, and contains black holes and Hawking radiation [1]. Starting from the seminal work of Callan, Giddings, Harvey and Strominger (CGHS) [4] where these properties were first realized, some interesting additional results about the CGHS model and its modifications [5-7] have been obtained (for a review, see [7]). In particular, 2d black holes — previously found in the string context [8] (for an earlier discussion, see [31]) - and their properties have been investigated intensively.

In Refs. $[9,11]$ black hole solutions have been obtained in $2 \mathrm{~d}$ dilaton-Maxwell gravity with the action

$$
S=-\int d^{2} x \sqrt{g}\left[\frac{1}{2} g^{\mu \nu} \nabla_{\mu} \Phi \nabla_{\nu} \Phi+C R \Phi+V(\Phi)+\frac{1}{4} f(\Phi) g^{\mu \alpha} g^{\nu \beta} F_{\mu \nu} F_{\alpha \beta}\right] .
$$

where $F_{\mu \nu}=\partial_{\mu} A_{\nu}-\partial_{\nu} A_{\mu}, \Phi$ is the dilaton, and $V(\Phi)$ and $f(\Phi)$ are the dilatonic and Maxwell potentials, respectively. Model (1) is connected (via some compactification) with the four-dimensional Einstein-Maxwell theory, which admits charged black hole solutions [10]. Particular cases of (1) describe the bosonic string effective action $\left(A_{\mu}=0\right)$ and the heterotic string effective action $\left(A_{\mu} \neq 0\right)$. The renormalization structure of $2 \mathrm{~d}$ dilaton gravity in covariant gauges has been investigated in Refs. [12-15]. It has been shown there that the theory is multiplicatively renormalizable off-shell for the Liuoville dilaton potential (for some specific choices of the parameters the theory is even finite [14]). Moreover, the one-loop on-shell effective action is given by surface counterterms only; that is why the one-loop $S$-matrix is finite, as in $4 d$ Einstein gravity [18]. This shows explicitly that $2 \mathrm{~d}$ quantum gravity can be a very good laboratory for studying the formal structure of quantum field theory. 
In the present paper we investigate the renormalization structure of $2 \mathrm{~d}$ dilaton-Maxwell gravity (1). In Sec. 2, we calculate the one-loop divergences of this convenient effective action in two covariant gauges (one of which is De Witt's gauge). All surface divergences are taken into account in this consideration. (Note that similar calculation in a simplest covariant gauge have been done in Refs. $[11,16])$. These terms may be important in the discussion of the Casimir effect [25]. In Sect. 3 we calculate the one-loop divergences in the unique effective action formalism $[19,20]$ (see [21] for a review), which gives a parametrization and gauge independent result. Finally, Sect. 4 is devoted to discussions and to a comparison of the results obtained in the two formalisms. In an Appendix we obtain the one-loop divergences of $2 \mathrm{~d}$ Yang-Mills dilaton gravity in the simplest covariant gauge.

\section{One-loop divergences of $2 \mathrm{~d}$ dilaton-Maxwell grav- ity in the convenient effective action formalism}

In this section we calculate the one-loop divergences (including surface divergences) of the convenient effective action (1) for 2 d dilaton-Maxwell gravity in the covariant gauge. The classical dynamics of the theory are defined by the following field equations

$$
\begin{aligned}
& \nabla_{\mu}\left(f F^{\mu \nu}\right)=0, \quad-\Delta \Phi+C R \Phi+V^{\prime}(\Phi)+\frac{1}{4} f^{\prime}(\Phi) F_{\mu \nu}^{2}=0, \\
& -\frac{1}{2}\left(\nabla^{\alpha} \Phi\right)\left(\nabla^{\beta} \Phi\right)+\frac{1}{4} g^{\alpha \beta} \nabla^{\mu} \Phi \nabla_{\mu} \Phi+C\left(\nabla^{\alpha} \nabla^{\beta}-g^{\alpha \beta} \Delta\right) \Phi \\
& +\frac{1}{2} g^{\alpha \beta} V+\frac{1}{8} g^{\alpha \beta} f F_{\mu \nu}^{2}-\frac{1}{2} f F_{\mu}^{\alpha} F^{\beta \mu}=0 .
\end{aligned}
$$

These equations wil be used in the discussion of the on-shell effective action.

The analysis of one-loop divergences is most conveniently carried out within the background field method. The fields are split into their quantum and background parts,

$$
\Phi \longrightarrow \bar{\Phi}=\Phi+\varphi, \quad A_{\mu} \longrightarrow \bar{A}_{\mu}=A_{\mu}+Q_{\mu}, \quad g_{\mu \nu} \longrightarrow \bar{g}_{\mu \nu}=g_{\mu \nu}+h_{\mu \nu},
$$

where the second terms $\varphi, Q_{\mu}$ and $h_{\mu \nu}$ are the quantum fields. In what follows we shall use the dynamical variables $h=g^{\mu \nu} h_{\mu \nu}$ and $\bar{h}_{\mu \nu}=h_{\mu \nu}-\frac{1}{2} h g_{\mu \nu}$, rather than $h_{\mu \nu}$.

In order to make contact with Ref. [15] — where the one-loop divergences (including the surface contributions) in the absence of the Maxwell terms have been calculated - we add to the action the following term

$$
\Delta S=-C \xi \int d^{2} x \sqrt{g}\left[\Phi \bar{h}_{\mu \nu}\left(R^{\mu \nu}-\frac{1}{2} R g^{\mu \nu}\right)\right],
$$


where $\xi$ is an arbitrary parameter. Owing to the 2 d identity $R_{\mu \nu}-\frac{1}{2} R g_{\mu \nu}=0$, expression (4) is obviously zero. The authors of ref.[15] do not have a clear interpretation of (4), which looks very similar to a kind of Wess-Zumino topological term. (However,they failed to find the classical counterpart for (4).) Notice that at any stage of the calculation, $\xi$ may be taken to be equal to zero. Notice also that the second variation of (4) may be important in order to represent the differential operator corresponding to the second variation of the classical action in a minimal form.Anyway,as we will see $\xi$ ( or more exactly $\gamma$ whatever its origin is) does not appear in renormalized effective action.

Let us now proceed with the construction of the De Witt covariant gauge, $\chi^{A}$, where $A=(*, \mu)$. This gauge will fix the $\mathrm{U}(1)$ transformations and the general covariant transformations

$$
\begin{array}{r}
\delta_{*} Q_{\mu}=-\nabla_{\mu} \omega^{*}, \quad \delta_{*} \varphi=\delta_{*} h=\delta_{*} \bar{h}_{\mu \nu}=0, \\
\delta Q_{\mu}=-\left(\nabla_{\nu} A_{\mu}\right) \omega^{\nu}-A_{\nu} \nabla_{\mu} \omega^{\nu}, \quad \delta h=-2 \nabla_{\mu} \omega^{\mu}, \\
\delta \bar{h}_{\mu \nu}=-\left(g_{\mu \lambda} \nabla_{\nu}+g_{\nu \lambda} \nabla_{\mu}-g_{\mu \nu} \nabla_{\lambda}\right) \omega^{\lambda}, \quad \delta \varphi=-\left(\nabla_{\mu} \Phi\right) \omega^{\lambda},
\end{array}
$$

where $\omega_{A}=\left(\omega^{*}, \omega^{\mu}\right)$ are parameters of the gauge transformations.

In order to construct the De Witt covariant gauge we need the metric on the space of fields $\varphi^{i}=\left(Q_{\mu}, \varphi, h, \bar{h}_{\mu \nu}\right)$ [24]. It is known that this metric contains an ambiguity in its definition. In particular, sometimes this metric is chosen to coincide with a matrix of higher derivatives of the quadratic expansion of the classical action [19]. (Notice that this ambiguity leads to a field space metric dependence of the unique effective action [23].) In the case under discussion the natural choice of this metric $G_{i j}$ is

$$
G_{i j}=\sqrt{g}\left(\begin{array}{cccc}
\Omega(\Phi) g^{\mu \nu} & 0 & 0 & 0 \\
0 & \Theta(\Phi) & C / 2 & 0 \\
0 & C / 2 & 0 & 0 \\
0 & 0 & 0 & \gamma \Phi P^{\mu \nu, \rho \sigma}
\end{array}\right)
$$

where $\gamma=\frac{C}{2}(\xi-1), P^{\mu \nu, \rho \sigma}=\delta^{\mu \nu, \rho \sigma}-\frac{1}{2} g^{\mu \nu} g^{\rho \sigma}$, and the functions $\Omega(\Phi)$ and $\Theta(\Phi)$ are arbitrary but sign preserving (this is the ambiguity in the definition of the configuration space metric $G_{i j}$ ).

The De Witt gauge is defined by the following condition

$$
\frac{\delta \chi^{A}}{\delta \varphi^{i}}=-\left(c^{-1}\right)^{A B} \nabla_{B}^{J} G_{i j}
$$

where the gauge group operators are

$$
\nabla_{*}^{Q_{\mu}}=\nabla_{\mu}, \quad \nabla_{\mu}^{Q_{\mu}}=F_{\mu \lambda}+A_{\lambda} \nabla_{\mu}
$$




$$
\nabla_{\mu}^{\varphi}=-\left(\nabla_{\mu} \Phi\right), \quad \nabla_{\mu}^{h}=2 \nabla_{\mu}, \quad \nabla_{\lambda}^{\bar{h}_{\mu \nu}}=2 g_{\lambda \mu} \nabla_{\nu}-g_{\mu \nu} \nabla_{\lambda}
$$

Notice that the symmmetry group is obviously not the direct product of the U(1) group and the group of general covariant transformations, although $\mathrm{U}(1)$ is certainly an invariant subgroup of it. Then, it is natural to choose the non-diagonal matrix

$$
c_{A B}=\frac{\Omega^{2}}{f} \sqrt{g}\left(\begin{array}{cc}
1 & A_{\nu} \\
A_{\mu} & A_{\mu} A_{\nu}+\frac{2 \gamma \Phi f}{\Omega^{2}} g_{\mu \nu}
\end{array}\right),
$$

which has a Kaluza-Klein structure [24]. (In other words, our choice of $c_{A B}$ leads to a natural embedding of both $g_{\mu \nu}$ and $A_{\nu}$ into a multidimensional metric.) Due to the exact dependence of $c_{A B}$ on $A_{\mu}$ this matrix is not covariant. However

$$
\operatorname{det} c_{A B}=\frac{2 \gamma \Phi \Omega^{2}}{f \sqrt{g}} .
$$

The inverse matrix is given by

$$
\left(c^{-1}\right)^{A B}=\frac{1}{2 \gamma \Phi \sqrt{g}}\left(\begin{array}{cc}
\frac{2 \gamma \Phi f}{\Omega^{2}}+A_{\lambda} A^{\lambda} & -A^{\nu} \\
-A^{\mu} & g^{\mu \nu}
\end{array}\right) .
$$

Finally, from (7), (8), (11), we find

$$
S_{G F}=-\frac{1}{2} \int d^{2} x c_{A B} \chi^{A} \chi^{B}
$$

where

$$
\begin{aligned}
\chi^{*}= & -\frac{f}{\Omega} \nabla^{\mu} Q_{\mu}-\frac{f}{\Omega^{2}} \Omega^{\prime}\left(\nabla^{\mu} \Phi\right) Q_{\mu}+\frac{\Omega}{2 \gamma \Phi} A_{\lambda} F^{\mu \lambda} Q_{\mu}-\frac{\Theta}{2 \gamma \Phi} A^{\lambda}\left(\nabla_{\lambda} \Phi\right) \varphi \\
& +\frac{C}{2 \gamma \Phi} A^{\lambda} \nabla_{\lambda} \varphi-\frac{C}{4 \gamma \Phi} A^{\lambda}\left(\nabla_{\lambda} \Phi\right) h+A^{\mu} \nabla^{\nu} \bar{h}_{\mu \nu}+\frac{1}{\Phi} A^{\mu}\left(\nabla^{\nu} \Phi\right) \bar{h}_{\mu \nu}, \\
\chi^{\mu}= & \frac{\Omega}{2 \gamma \Phi} F^{\mu \lambda} Q_{\lambda}-\frac{C}{2 \gamma \Phi} \nabla^{\mu} \varphi+\frac{\Theta}{2 \gamma \Phi}\left(\nabla^{\mu} \Phi\right) \varphi+\frac{C}{4 \gamma \Phi}\left(\nabla^{\mu} \Phi\right) h \\
& -\nabla^{\nu} \bar{h}_{\mu \nu}-\frac{1}{\Phi}\left(\nabla^{\nu} \Phi\right) \bar{h}_{\nu}^{\mu} .
\end{aligned}
$$

Now the De Witt gauge appears to be minimal. After some tedious algebra, the total quadratic expansion of the classical action can be calculated

$$
\begin{aligned}
S_{t o t}^{(2)} & =S^{(2)}+\Delta S^{(2)}+S_{G F} \\
& =-\frac{1}{2} \int d^{2} x \sqrt{g}\left\{Q _ { \mu } \left[-f g^{\mu \alpha} \Delta+\left(\frac{f \Omega^{\prime}}{\Omega}-f^{\prime}\right)\left(\nabla^{\mu} \Phi\right) \nabla^{\alpha}-\left(\frac{f \Omega^{\prime}}{\Omega}-f^{\prime}\right)\left(\nabla^{\alpha} \Phi\right) \nabla^{\mu}\right.\right.
\end{aligned}
$$




$$
\begin{aligned}
& +\left(2 \frac{f \Omega^{\prime 2}}{\Omega^{2}}-\frac{f^{\prime} \Omega^{\prime}}{\Omega}-\frac{f \Omega^{\prime \prime}}{\Omega}\right)\left(\nabla^{\mu} \Phi\right)\left(\nabla^{\alpha} \Phi\right)+\frac{1}{2} f^{\prime} g^{\mu \alpha}(\Delta \Phi) \\
& \left.+\frac{1}{2} f R g^{\mu \alpha}+\frac{1}{2} f^{\prime \prime} g^{\mu \alpha}\left(\nabla^{\lambda} \Phi\right)\left(\nabla_{\lambda} \Phi\right)-\frac{f \Omega^{\prime}}{\Omega}\left(\nabla^{\mu} \nabla^{\alpha} \Phi\right)+\frac{\Omega^{2}}{2 \gamma \Phi} F_{\lambda}{ }^{\mu} F^{\lambda \alpha}\right] Q_{\alpha} \\
& +Q_{\mu}\left[\left(\frac{\Omega C}{2 \gamma \Phi}+f^{\prime}\right) F^{\mu \lambda} \nabla_{\lambda}+\left(\frac{f^{\prime}}{2}-\frac{\Omega C}{4 \gamma \Phi}\right)\left(\nabla_{\lambda} F^{\mu \lambda}\right)\right. \\
& \left.+\left(\frac{f^{\prime \prime}}{2}+\frac{\Omega C}{4 \gamma \Phi^{2}}-\frac{C \Omega^{\prime}}{4 \gamma \Phi}-\frac{\Omega \Theta}{2 \gamma \Phi}\right) F^{\mu \lambda}\left(\nabla_{\lambda} \Phi\right)\right] \varphi \\
& +\varphi\left[-\left(\frac{\Omega C}{2 \gamma \Phi}+f^{\prime}\right) F^{\alpha \lambda} \nabla_{\lambda}+\left(\frac{f^{\prime}}{2}-\frac{\Omega C}{4 \gamma \Phi}\right)\left(\nabla_{\lambda} F^{\alpha \lambda}\right)\right. \\
& \left.+\left(\frac{f^{\prime \prime}}{2}+\frac{\Omega C}{4 \gamma \Phi^{2}}-\frac{C \Omega^{\prime}}{4 \gamma \Phi}-\frac{\Omega \Theta}{2 \gamma \Phi}\right) F^{\alpha \lambda}\left(\nabla_{\lambda} \Phi\right)\right] Q_{\alpha} \\
& +Q_{\mu}\left[-\frac{1}{2} f F^{\mu \lambda} \nabla_{\lambda}-\frac{1}{4} f\left(\nabla_{\lambda} F^{\mu \lambda}\right)-\left(\frac{1}{4} f^{\prime}+\frac{\Omega C}{4 \gamma \Phi}\right) F^{\mu \lambda}\left(\nabla_{\lambda} \Phi\right)\right] h \\
& +h\left[\frac{1}{2} f F^{\alpha \lambda} \nabla_{\lambda}-\frac{1}{4} f\left(\nabla_{\lambda} F^{\alpha \lambda}\right)-\left(\frac{1}{4} f^{\prime}+\frac{\Omega C}{4 \gamma \Phi}\right) F^{\alpha \lambda}\left(\nabla_{\lambda} \Phi\right)\right] Q_{\alpha} \\
& +Q_{\mu}\left[(\Omega-f) F^{\mu \alpha} \nabla^{\beta}-f g^{\mu \beta} F^{\alpha \lambda} \nabla_{\lambda}-\frac{1}{2}(f+\Omega)\left(\nabla^{\beta} F^{\mu \alpha}\right)\right. \\
& +\left(\frac{\Omega}{\Phi}-\frac{\Omega^{\prime}}{2}-\frac{1}{2} f^{\prime}\right) F^{\mu \alpha}\left(\nabla^{\beta} \Phi\right) \\
& \left.-\frac{1}{2} f g^{\mu \beta}\left(\nabla_{\lambda} F^{\alpha \lambda}\right)-\frac{1}{2} f^{\prime} g^{\mu \beta} F^{\alpha \lambda}\left(\nabla_{\lambda} \Phi\right)\right] \bar{h}_{\alpha \beta} \\
& +\bar{h}_{\mu \nu}\left[-(\Omega-f) F^{\alpha \mu} \nabla^{\nu}+f g^{\nu \alpha} F^{\mu \lambda} \nabla_{\lambda}-\frac{1}{2}(f+\Omega)\left(\nabla^{\nu} F^{\alpha \mu}\right)\right. \\
& +\left(+\frac{\Omega}{\Phi}-\frac{\Omega^{\prime}}{2}-\frac{1}{2} f^{\prime}\right) F^{\alpha \mu}\left(\nabla^{\nu} \Phi\right) \\
& \left.-\frac{1}{2} f g^{\nu \alpha}\left(\nabla_{\lambda} F^{\mu \lambda}\right)-\frac{1}{2} f^{\prime} g^{\nu \alpha} F^{\mu \lambda}\left(\nabla_{\lambda} \Phi\right)\right] Q_{\alpha} \\
& +\varphi\left[-\left(1+\frac{C^{2}}{2 \gamma \Phi}\right) \Delta+\left(\frac{C \Theta}{2 \gamma \Phi}-\frac{C^{2}}{4 \gamma \Phi^{2}}\right)(\Delta \Phi)\right. \\
& \left.+\left(\frac{C \Theta^{\prime}}{2 \gamma \Phi}-\frac{C \Theta}{2 \gamma \Phi^{2}}+\frac{C^{2}}{2 \gamma \Phi^{3}}+\frac{\Theta^{2}}{2 \gamma \Phi}\right)\left(\nabla_{\lambda} \Phi\right)\left(\nabla^{\lambda} \Phi\right)+V^{\prime \prime}+\frac{1}{4} f^{\prime \prime} F^{2}\right] \varphi
\end{aligned}
$$




$$
\begin{aligned}
& +h\left[\frac{C}{4}(\Delta \Phi)+\frac{1}{8} f F^{2}+\frac{C^{2}}{8 \gamma \Phi}\left(\nabla^{\lambda} \Phi\right)\left(\nabla_{\lambda} \Phi\right)\right] h \\
& +h\left[-\frac{C}{2} \Delta-\frac{C^{2}}{4 \gamma \Phi}\left(\nabla^{\lambda} \Phi\right) \nabla_{\lambda}+\left(\frac{C \Theta}{4 \gamma \Phi}-\frac{C^{2}}{8 \gamma \Phi^{2}}\right)\left(\nabla^{\lambda} \Phi\right)\left(\nabla_{\lambda} \Phi\right)\right. \\
& \left.+\frac{C^{2}}{8 \gamma \Phi}(\Delta \Phi)+\frac{1}{2} V^{\prime}-\frac{1}{8} f^{\prime} F^{2}\right] \varphi \\
& +\varphi\left[-\frac{C}{2} \Delta+\frac{C^{2}}{4 \gamma \Phi}\left(\nabla^{\lambda} \Phi\right) \nabla_{\lambda}+\left(\frac{C \Theta}{4 \gamma \Phi}-\frac{C^{2}}{8 \gamma \Phi^{2}}\right)\left(\nabla^{\lambda} \Phi\right)\left(\nabla_{\lambda} \Phi\right)\right. \\
& \left.+\frac{C^{2}}{8 \gamma \Phi}(\Delta \Phi)+\frac{1}{2} V^{\prime}-\frac{1}{8} f^{\prime} F^{2}\right] h \\
& +\bar{h}_{\mu \nu}\left[\left(\Theta+\frac{C}{\Phi}-1\right)\left(\nabla^{\mu} \Phi\right) \nabla^{\nu}+\left(\frac{C}{2 \Phi^{2}}-\frac{\Theta}{\Phi}+\frac{\Theta^{\prime}}{2}\right)\left(\nabla^{\mu} \Phi\right)\left(\nabla^{\nu} \Phi\right)\right. \\
& \left.+\left(\frac{\Theta+1}{2}-\frac{C}{2 \Phi}\right)\left(\nabla^{\mu} \nabla^{\nu} \Phi\right)-\frac{1}{2} f^{\prime} F_{\lambda}^{\mu} F^{\nu \lambda}\right] \varphi \\
& +\varphi\left[-\left(\Theta+\frac{C}{\Phi}-1\right)\left(\nabla^{\alpha} \Phi\right) \nabla^{\beta}+\left(\frac{C}{2 \Phi^{2}}-\frac{\Theta}{\Phi}+\frac{\Theta^{\prime}}{2}\right)\left(\nabla^{\alpha} \Phi\right)\left(\nabla^{\beta} \Phi\right)\right. \\
& \left.+\left(\frac{\Theta+1}{2}-\frac{C}{2 \Phi}\right)\left(\nabla^{\alpha} \nabla^{\beta} \Phi\right)-\frac{1}{2} f^{\prime} F_{\lambda}^{\alpha} F^{\beta \lambda}\right] \bar{h}_{\alpha \beta} \\
& +\bar{h}_{\mu \nu}\left[\left(\frac{1}{4}-\frac{C}{2 \gamma \Phi}\right)\left(\nabla^{\mu} \Phi\right)\left(\nabla^{\nu} \Phi\right)+\frac{1}{2} f F_{\lambda}^{\mu} F^{\nu \lambda}\right] h \\
& +h\left[\left(\frac{1}{4}-\frac{C}{2 \gamma \Phi}\right)\left(\nabla^{\alpha} \Phi\right)\left(\nabla^{\beta} \Phi\right)+\frac{1}{2} f F_{\lambda}^{\alpha} F^{\beta \lambda}\right] \bar{h}_{\alpha \beta} \\
& +\bar{h}_{\mu \nu}\left[-\gamma \Phi \delta^{\mu \nu, \alpha \beta} \Delta+\left(\gamma+\frac{C}{2}\right) g^{\nu \beta}\left(\nabla^{\mu} \Phi\right) \nabla^{\alpha}\right. \\
& -\left(\gamma+\frac{C}{2}\right) g^{\nu \beta}\left(\nabla^{\alpha} \Phi\right) \nabla^{\mu}-\left(\frac{3}{2} C+\gamma\right) g^{\nu \beta}\left(\nabla^{\mu} \nabla^{\alpha} \Phi\right) \\
& +\frac{3}{4} C(\Delta \Phi) \delta^{\mu \nu, \alpha \beta}-\frac{1}{4} \delta^{\mu \nu, \alpha \beta}\left(\nabla_{\lambda} \Phi\right)\left(\nabla^{\lambda} \Phi\right) \\
& +\left(1+\frac{2 \gamma}{\Phi}\right) g^{\nu \beta}\left(\nabla^{\mu} \Phi\right)\left(\nabla^{\alpha} \Phi\right)+\gamma \Phi R \delta^{\mu \nu, \alpha \beta}-\frac{1}{2} V \delta^{\mu \nu, \alpha \beta} \\
& \left.\left.-\frac{1}{8} f F^{2} \delta^{\mu \nu, \alpha \beta}+g^{\nu \beta} f F_{\lambda}^{\mu} F^{\alpha \lambda}+\frac{1}{2} f F^{\mu \alpha} F^{\nu \beta}\right] \bar{h}_{\alpha \beta}\right\} .
\end{aligned}
$$

As is evident from (14), the total quadratic expansion of the action can be written as 
follows

$$
-\frac{1}{2} \varphi^{i} \hat{H} \varphi^{j} \equiv-\frac{1}{2} \varphi^{i}\left[\hat{K}_{i j} \Delta+\hat{L}_{\lambda, i j} \nabla^{\lambda}+\hat{M}_{i j}\right] \varphi^{j}
$$

where the explicit form of the operator $\hat{H}$ can readily be read off from Eq. (14). However, the extra integrations by parts change the matrix elements of the operator $\hat{H}$ and can destroy its desired properties (properly symmetrized, $\hat{H}$ should be hermitean). In order to have this operator uniquely defined, the doubling trick by 't Hooft and Veltman [18] is very useful. A clear explanation of how to apply it in the present context can be found in Refs. [13,14]. In fact, using this method amounts to the following redefinitions of the operators in $\hat{H}(15)$ :

$$
\begin{gathered}
\hat{H} \rightarrow \hat{H}^{\prime}=-\hat{K} \Delta+\hat{L}_{\lambda}^{\prime} \nabla^{\lambda}+\hat{M}^{\prime}, \\
\hat{L}_{\lambda}^{\prime}=\frac{1}{2}\left(\hat{L}_{\lambda}-\hat{L}_{\lambda}^{T}\right)-\nabla^{\lambda} \hat{K}, \\
\hat{M}^{\prime}=\frac{1}{2}\left(\hat{M}+\hat{M}^{T}\right)-\frac{1}{2} \nabla^{\lambda} \hat{L}_{\lambda}^{T}-\frac{1}{2} \Delta \hat{K},
\end{gathered}
$$

where the operators of $\hat{H}^{\prime}$ are given by

$$
\hat{K}_{i j}=\left.\hat{G}_{i j}\right|_{\Theta \rightarrow 1+C^{2} / 2 \gamma \Phi}, \quad\left(\hat{K}^{-1}\right)^{i j}=\frac{1}{\sqrt{g}}\left(\begin{array}{cccc}
\frac{1}{f} g_{\mu \alpha} & 0 & 0 & 0 \\
0 & 0 & \frac{2}{C} & 0 \\
0 & \frac{2}{C} & -\left(\frac{4}{C^{2}}+\frac{2}{\gamma \Phi}\right) & 0 \\
0 & 0 & 0 & \frac{1}{\gamma \Phi} P_{\mu \nu, \alpha \beta}
\end{array}\right),
$$

and

$$
\begin{aligned}
& \hat{L}_{11}^{\prime \lambda}=\frac{f \Omega^{\prime}-f^{\prime} \Omega}{\Omega}\left[\left(\nabla^{\mu} \Phi\right) g^{\alpha \lambda}-\left(\nabla^{\alpha} \Phi\right) g^{\mu \lambda}\right]-f^{\prime}\left(\nabla^{\lambda} \Phi\right) g^{\mu \alpha} \\
& \hat{L}_{12}^{\prime \lambda}=-\hat{L}_{21}^{\lambda}=\left(f^{\prime}+\frac{\Omega C}{2 \gamma \Phi}\right) F^{\mu \lambda} ; \\
& \hat{L}_{13}^{\prime \lambda}=-\hat{L}_{31}^{\lambda}=-\frac{1}{2} f F^{\mu \lambda} ; \\
& \hat{L}_{14}^{\prime \lambda}=-\hat{L}_{41}^{\lambda}=f F_{\omega}^{\lambda} P^{\alpha \beta, \mu \omega}+(\Omega-f) F_{\omega}^{\mu} P^{\alpha \beta, \lambda \omega} ; \\
& \hat{L}_{22}^{\prime \lambda}=\left(\frac{C^{2}}{2 \gamma \Phi^{2}}-\frac{C \Theta}{\gamma \Phi}\right)\left(\nabla^{\lambda} \Phi\right) ; \\
& \hat{L}_{23}^{\prime \lambda}=-\hat{L}_{32}^{\prime \lambda}=\frac{C^{2}}{4 \gamma \Phi}\left(\nabla^{\lambda} \Phi\right) ; \\
& \hat{L}_{24}^{\prime \lambda}=-\hat{L}_{42}^{\prime \lambda}=\left(1-\Theta-\frac{C}{\Phi}\right)\left(\nabla_{\omega} \Phi\right) P^{\alpha \beta, \lambda \omega} ;
\end{aligned}
$$




$$
\begin{aligned}
\hat{L}_{33}^{\prime \lambda}= & -\frac{C}{2}\left(\nabla^{\lambda} \Phi\right) ; \\
\hat{L}_{34}^{\prime \lambda}= & -\hat{L}_{43}^{\prime \lambda}=0 ; \\
\hat{L}_{44}^{\prime \lambda}= & \left(\frac{C}{2}+\gamma\right)\left(\nabla^{\omega} \Phi\right)\left[P_{\omega \kappa}^{\mu \nu} P^{\alpha \beta, \lambda \kappa}-P^{\mu \nu, \lambda \kappa} P_{\omega \kappa}^{\alpha \beta}\right]-\frac{3 C}{2}\left(\nabla^{\lambda} \Phi\right) P^{\mu \nu, \alpha \beta} ; \\
\hat{M}_{11}^{\prime}= & \frac{1}{2} f R g^{\mu \alpha}+\left(2 \frac{f \Omega^{\prime 2}}{\Omega^{2}}-\frac{f^{\prime} \Omega^{\prime}}{\Omega}-\frac{f \Omega^{\prime \prime}}{\Omega}\right)\left(\nabla^{\mu} \Phi\right)\left(\nabla^{\alpha} \Phi\right) \\
& -\frac{f \Omega^{\prime}}{\Omega}\left(\nabla^{\mu} \nabla^{\alpha} \Phi\right)+\frac{\Omega^{2}}{2 \gamma \Phi} F_{\lambda}^{\mu} F_{\lambda \alpha} ; \\
\hat{M}_{23}^{\prime}= & \hat{M}_{32}^{\prime}=\left(\frac{C \Theta}{4 \gamma \Phi}-\frac{C^{2}}{8 \gamma \Phi^{2}}\right)\left(\nabla^{\lambda} \Phi\right)\left(\nabla_{\lambda} \Phi\right)+\frac{C^{2}}{8 \gamma \Phi}(\Delta \Phi)+\frac{1}{2} V^{\prime}-\frac{1}{8} f^{\prime} F^{2} ; \\
\hat{M}_{33}^{\prime}= & \frac{C^{2}}{8 \gamma \Phi}\left(\nabla^{\lambda} \Phi\right)\left(\nabla_{\lambda} \Phi\right)+\frac{1}{8} f F^{2} ; \\
\hat{M}_{44}^{\prime}= & {\left[\left(1+\frac{2 \gamma}{\Phi}\right)\left(\nabla^{\lambda} \Phi\right)\left(\nabla_{\omega} \Phi\right)-\left(\gamma+\frac{3 C}{2}\right)\left(\nabla^{\lambda} \nabla_{\omega} \Phi\right)+f F_{\omega \rho} F^{\lambda \rho}\right] P^{\mu \nu, \omega \kappa} P_{\lambda \kappa}^{\alpha \beta} } \\
& +\left[-\frac{1}{4}\left(\nabla^{\lambda} \Phi\right)\left(\nabla_{\lambda} \Phi\right)+\gamma \Phi R-\frac{1}{2} V-\frac{1}{8} f F^{2}\right] P^{\mu \nu, \alpha \beta}+\frac{1}{2} f F^{\omega \lambda} F^{\kappa \rho} P_{\omega \kappa}^{\mu \nu} P_{\lambda \rho}^{\alpha \beta}(18)
\end{aligned}
$$

The other components of $\hat{M}^{\prime}$ are not essential for us since they do not contribute to the divergencies of the efective action $\Gamma$.

Introducing the notations $\hat{E}^{\lambda}=-(1 / 2) \hat{K}^{-1} \hat{L}^{\prime \lambda}$ and $\hat{\Pi}=-\hat{K}^{-1} \hat{M}^{\prime}$, the operator $\hat{H}^{\prime}$ can be put in the form

$$
\hat{H}^{\prime}=-\hat{K}\left(\hat{1} \Delta+2 \hat{E}^{\lambda} \nabla_{\lambda}+\hat{\Pi}\right) .
$$

The one-loop gravitational-Maxwell contribution to the effective action is given by the standard expression:

$$
\begin{aligned}
\Gamma_{d i v} & =\left.\frac{i}{2} \operatorname{Tr} \ln \hat{H}^{\prime}\right|_{d i v}=\left.\frac{i}{2} \operatorname{Tr} \ln \left(\hat{1} \Delta+2 \hat{E}^{\lambda} \nabla_{\lambda}+\hat{\Pi}\right)\right|_{d i v} \\
& =\frac{1}{2 \epsilon} \int d^{2} x \operatorname{Tr}\left[\hat{\Pi}+\frac{R}{6} \hat{1}-\hat{E}^{\lambda} \hat{E}_{\lambda}-\nabla_{\lambda} \hat{E}^{\lambda}\right]
\end{aligned}
$$

where $\epsilon=2 \pi(n-2)$ and $\operatorname{Tr} \ln (-\hat{K})$ gives a contribution proportional to $\delta(0)$, which is zero in dimensional regularization. Notice that the term $-\nabla_{\lambda} \hat{E}^{\lambda}$ is missing in the algorithm (20) corresponding to Ref. [15]. This will lead to some disagreement in the surface terms corresponding to the pure dilatonic sector, as compared with Ref. [15]. 
The components of $\hat{E}^{\lambda}$ and $\hat{\Pi}$ can be easily evaluated from (18):

$$
\begin{aligned}
& \left(\hat{E}^{\lambda}\right)_{1}^{1}=\frac{f \Omega^{\prime}-f^{\prime} \Omega}{2 f \Omega}\left[\left(\nabla^{\alpha} \Phi\right) g_{\rho}^{\lambda}-\left(\nabla_{\rho} \Phi\right) g^{\alpha \lambda}\right]+\frac{f^{\prime}}{2 f}\left(\nabla^{\lambda} \Phi\right) g_{\rho}^{\alpha} ; \\
& \left(\hat{E}^{\lambda}\right)_{2}^{1}=-\left(\frac{f^{\prime}}{2 f}+\frac{\Omega C}{4 \gamma \Phi f}\right) F_{\rho}^{\lambda} \\
& \left(\hat{E}^{\lambda}\right)_{3}^{1}=\frac{1}{4} F_{\rho}^{\lambda} \\
& \left(\hat{E}^{\lambda}\right)_{4}^{1}=\frac{f-\Omega}{2 f} F_{\rho \omega} P^{\alpha \beta, \rho \omega}-\frac{1}{2} F^{\lambda \omega} P_{\rho \omega}^{\alpha \beta} ; \\
& \left(\hat{E}^{\lambda}\right)_{1}^{2}=-\frac{f}{2 C} F^{\alpha \lambda} \\
& \left(\hat{E}^{\lambda}\right)_{2}^{2}=\frac{C}{4 \gamma \Phi}\left(\nabla^{\lambda} \Phi\right) \\
& \left(\hat{E}^{\lambda}\right)_{3}^{2}=\left(\hat{E}^{\lambda}\right)_{4}^{2}=0 \\
& \left(\hat{E}^{\lambda}\right)_{1}^{3}=\left(\frac{f}{C^{2}}+\frac{f+\Omega}{2 \gamma \Phi}+\frac{f^{\prime}}{C}\right) F^{\alpha \lambda} \\
& \left(\hat{E}^{\lambda}\right)_{2}^{3}=-\left(\frac{1}{2 \gamma \Phi}+\frac{C}{2 \gamma \Phi^{2}}+\frac{C^{2}}{4 \gamma^{2} \Phi^{2}}\right)\left(\nabla^{\lambda} \Phi\right) \text {; } \\
& \left(\hat{E}^{\lambda}\right)_{3}^{3}=-\frac{C}{4 \gamma \Phi}\left(\nabla^{\lambda} \Phi\right) \text {; } \\
& \left(\hat{E}^{\lambda}\right)_{4}^{3}=\left(\frac{\Theta-1}{C}+\frac{1}{\Phi}\right)\left(\nabla_{\omega} \Phi\right) P^{\alpha \beta, \lambda \omega} ; \\
& \left(\hat{E}^{\lambda}\right)_{1}^{4}=\frac{f}{2 \gamma \Phi} F_{\omega}^{\lambda} P_{\rho \sigma}^{\alpha \omega}+\frac{\Omega-f}{2 \gamma \Phi} F_{\omega}^{\alpha} P_{\rho \sigma}^{\lambda \omega} ; \\
& \left(\hat{E}^{\lambda}\right)_{2}^{4}=\left(\frac{1-\Theta}{2 \gamma \Phi}-\frac{C}{2 \gamma \Phi^{2}}\right)\left(\nabla_{\omega} \Phi\right) P_{\rho \sigma}^{\lambda \omega} \\
& \left(\hat{E}^{\lambda}\right)_{3}^{4}=0 \text {; } \\
& \left(\hat{E}^{\lambda}\right)_{4}^{4}=\left(\frac{1}{2 \Phi}+\frac{C}{4 \gamma \Phi}\right)\left(\nabla^{\omega} \Phi\right)\left[P_{\rho \sigma}^{\lambda \kappa} P_{\omega \kappa}^{\alpha \beta}-P_{\rho \sigma, \omega \kappa} P^{\alpha \beta, \lambda \kappa}\right]+\frac{1}{2 \gamma \Phi}\left(\nabla^{\lambda} \Phi\right) P_{\rho \sigma}^{\alpha \beta} ; \\
& \hat{\Pi}_{1}^{1}=-\frac{1}{2} R g_{\alpha}^{\rho}+\left(\frac{\Omega^{\prime \prime}}{\Omega}+\frac{f^{\prime} \Omega^{\prime}}{f \Omega}-\frac{2 \Omega^{\prime 2}}{\Omega^{2}}\right)\left(\nabla_{\rho} \Phi\right)\left(\nabla^{\alpha} \Phi\right)+\frac{\Omega^{\prime}}{\Omega}\left(\nabla_{\rho} \nabla^{\alpha} \Phi\right)-\frac{\Omega^{2}}{2 \gamma \Phi} F_{\rho \lambda} F^{\alpha \lambda} ;
\end{aligned}
$$




$$
\begin{aligned}
\hat{\Pi}_{2}^{2}= & -\frac{\Theta}{2 \gamma \Phi}\left(\nabla^{\lambda} \Phi\right)\left(\nabla_{\lambda} \Phi\right)-\frac{1}{C} V^{\prime}+\frac{f^{\prime}}{4 C} F^{2} ; \\
\hat{\Pi}_{3}^{3}= & \left(\frac{1-\Theta}{2 \gamma \Phi}+\frac{C}{2 \gamma \Phi^{2}}+\frac{C^{2}}{4 \gamma^{2} \Phi^{2}}\right)\left(\nabla^{\lambda} \Phi\right)\left(\nabla_{\lambda} \Phi\right)+\frac{1}{C}(\Delta \Phi)-\frac{1}{C} V^{\prime} \\
& +\left(\frac{f}{2 C^{2}}+\frac{f}{4 \gamma \Phi}+\frac{f^{\prime}}{4 C}\right) F^{2} ; \\
\hat{\Pi}_{4}^{4}= & {\left[\left(\frac{3 C}{2 \gamma \Phi}+\frac{1}{\Phi}\right)\left(\nabla^{\lambda} \nabla_{\omega} \Phi\right)-\left(\frac{1}{\gamma \Phi}+\frac{2}{\Phi^{2}}\right)\left(\nabla^{\lambda} \Phi\right)\left(\nabla_{\omega} \Phi\right)-\frac{f}{\gamma \Phi} F_{\omega \nu} F^{\lambda \nu}\right] P_{\rho \sigma}^{\omega \kappa} P_{\lambda \kappa}^{\alpha \beta} } \\
& +\left[\left(\frac{1}{2 \Phi}-\frac{3 C}{4 \gamma \Phi}\right)(\Delta \Phi)+\frac{1}{4 \gamma \Phi}\left(\nabla^{\lambda} \Phi\right)\left(\nabla_{\lambda} \Phi\right)-R+\frac{1}{2 \gamma \Phi} V+\frac{f}{8 \gamma \Phi} F^{2}\right] P_{\rho \sigma}^{\alpha \beta} \\
& -\frac{f}{2 \gamma \Phi} F^{\omega \kappa} F^{\lambda \nu} P_{\rho \sigma, \omega \lambda} P_{\kappa \nu}^{\alpha \beta} .
\end{aligned}
$$

Now, it is straightforward to calculate

$$
\begin{aligned}
& \operatorname{Tr}\left(\nabla_{\lambda} \hat{E}^{\lambda}\right)= \nabla_{\lambda}\left[\left(\frac{f^{\prime}}{f}+\frac{1}{\Phi}\right)\left(\nabla^{\lambda} \Phi\right)\right] \\
&=\left(\frac{f^{\prime \prime}}{f}-\frac{f^{\prime 2}}{f^{2}}-\frac{1}{\Phi^{2}}\right)\left(\nabla^{\lambda} \Phi\right)\left(\nabla_{\lambda} \Phi\right)+\left(\frac{f^{\prime}}{f}+\frac{1}{\Phi}\right)(\Delta \Phi) ; \\
& \operatorname{Tr}\left(\hat{E}^{\lambda} \hat{E}_{\lambda}\right)=\left(\frac{f^{\prime} \Omega^{\prime}}{f \Omega}-\frac{\Omega^{\prime 2}}{2 \Omega^{2}}-\frac{C}{2 \gamma \Phi^{2}}\right)\left(\nabla^{\lambda} \Phi\right)\left(\nabla_{\lambda} \Phi\right) \\
&+\left(\frac{f^{\prime}}{C}+\frac{f}{2 C^{2}}+\frac{f}{4 \gamma \Phi}+\frac{\Omega}{4 \gamma \Phi}-\frac{\Omega^{2}}{2 \gamma \Phi f}\right) F^{2} ; \\
& \operatorname{Tr}\left(\frac{R}{6} \hat{1}+\hat{\Pi}\right)=-2 R-\frac{2}{C} V^{\prime}+\frac{1}{\gamma \Phi} V+\left(\frac{f^{\prime}}{2 C}+\frac{f}{2 C^{2}}-\frac{\Omega^{2}}{2 \gamma \Phi f}\right) F^{2} \\
&+\left(\frac{\Omega^{\prime}}{\Omega}+\frac{1}{C}+\frac{2}{\Phi}\right)(\Delta \Phi) \\
&+\left(\frac{\Omega^{\prime \prime}}{\Omega}+\frac{f^{\prime} \Omega^{\prime}}{f \Omega}-\frac{2 \Omega^{\prime 2}}{\Omega^{2}}-\frac{\Theta}{\gamma \Phi}-\frac{2}{\Phi^{2}}+\frac{C}{2 \gamma \Phi^{2}}+\frac{C^{2}}{4 \gamma^{2} \Phi^{2}}\right)\left(\nabla^{\lambda} \Phi\right)\left(\nabla_{\lambda} \Phi\right) .
\end{aligned}
$$

In absence of background vectors $\left(F^{2}=0\right)$, Eq. (22) coincides with the corresponding expression in [15] (but for not the $\nabla_{\lambda} \hat{E}^{\lambda}$ term, which has been missed in [15]). Finally, from (20) and (22), we get

$$
\Gamma_{G M, d i v}=-\frac{1}{2 \epsilon} \int d^{2} x \sqrt{g}\left\{2 R-\frac{1}{\gamma \Phi} V+\frac{2}{C} V^{\prime}+\left[\frac{f^{\prime}}{2 C}+\frac{f}{4 \gamma \Phi}+\frac{\Omega}{2 \gamma \Phi}\right] F^{2}\right.
$$




$$
\begin{aligned}
& +\left[\frac{f^{\prime}}{f}-\frac{\Omega^{\prime}}{\Omega}-\frac{1}{C}-\frac{1}{\Phi}\right](\Delta \Phi) \\
& \left.+\left[\frac{3 \Omega^{\prime 2}}{2 \Omega^{2}}-\frac{\Omega^{\prime \prime}}{\Omega}-\frac{f^{\prime 2}}{f^{2}}+\frac{f^{\prime \prime}}{f}+\frac{\Theta}{\gamma \Phi}+\frac{1}{\Phi^{2}}-\frac{C}{\gamma \Phi^{2}}-\frac{C^{2}}{4 \gamma^{2} \Phi^{2}}\right]\left(\nabla^{\lambda} \Phi\right)\left(\nabla_{\lambda} \Phi\right)\right\} .
\end{aligned}
$$

In order to complete this calculation we must take into account the ghost contribution. The ghost operator $\hat{M}_{g h}$ is defined as

$$
\hat{M}_{g h B}^{A}=\nabla_{B}^{j} \frac{\delta \chi^{A}}{\delta \varphi^{j}} .
$$

It has for components

$$
\begin{aligned}
& \hat{M}_{*}^{*}=\frac{f}{\Omega} \Delta+\frac{f \Omega^{\prime}}{\Omega^{2}}\left(\nabla^{\lambda} \Phi\right) \nabla_{\lambda}+\frac{\Omega}{2 \gamma \Phi} A_{\mu} F^{\mu \lambda} \nabla_{\lambda}, \\
& \hat{M}_{*}^{\mu}=-\frac{\Omega}{2 \gamma \Phi} F^{\mu \lambda} \nabla_{\lambda} \\
& \hat{M}_{\nu}^{*}=\frac{f-\Omega}{\Omega} A_{\nu} \Delta+\left(\frac{f \Omega^{\prime}}{\Omega^{2}}-\frac{1}{\Phi}\right) A_{\nu}\left(\nabla^{\lambda} \Phi\right) \nabla_{\lambda}+\frac{\Omega}{2 \gamma \Phi} A_{\mu} A_{\nu} F^{\mu \lambda} \nabla_{\lambda} \\
& +\frac{f}{\Omega}\left(\nabla_{\nu} A_{\lambda}\right) \nabla^{\lambda}+\frac{f}{\Omega}\left(\nabla_{\lambda} A_{\nu}\right) \nabla^{\lambda}+\left(\frac{C}{2 \gamma \Phi}+\frac{1}{\Phi}\right) A_{\lambda}\left(\nabla^{\lambda} \Phi\right) \nabla_{\nu} \\
& -\left(\frac{C}{2 \gamma \Phi}+\frac{1}{\Phi}\right) A^{\lambda}\left(\nabla_{\nu} \Phi\right) \nabla_{\lambda}+\frac{f-\Omega}{2 \Omega} R A_{\nu}+\frac{f}{\Omega}\left(\nabla_{\nu} \nabla^{\lambda} A_{\lambda}\right) \\
& +\frac{\Theta}{2 \gamma \Phi} A_{\lambda}\left(\nabla^{\lambda} \Phi\right)\left(\nabla_{\nu} \Phi\right)-\frac{C}{2 \gamma \Phi} A_{\lambda}\left(\nabla^{\lambda} \nabla_{\nu} \Phi\right)+\frac{f \Omega^{\prime}}{\Omega^{2}}\left(\nabla^{\lambda} \Phi\right)\left(\nabla_{\nu} A_{\lambda}\right) \\
& +\frac{\Omega}{2 \gamma \Phi} A_{\mu} F^{\mu \lambda}\left(\nabla_{\nu} A_{\lambda}\right) \\
& \hat{M}_{\nu}^{\mu}=\delta_{\nu}^{\mu} \Delta+\frac{1}{\Phi} \delta_{\nu}^{\mu}\left(\nabla_{\lambda} \Phi\right) \nabla^{\lambda}-\frac{\Omega}{2 \gamma \Phi} A_{\nu} F^{\mu \lambda} \nabla^{\lambda}+\left(\frac{C}{2 \gamma \Phi}+\frac{1}{\Phi}\right)\left(\nabla_{\nu} \Phi\right) \nabla^{\mu} \\
& -\left(\frac{C}{2 \gamma \Phi}+\frac{1}{\Phi}\right)\left(\nabla^{\mu} \Phi\right) \nabla_{\nu}+\frac{R}{2} \delta_{\nu}^{\mu}+\frac{C}{2 \gamma \Phi}\left(\nabla^{\mu} \nabla_{\nu} \Phi\right) \\
& -\frac{\Theta}{2 \gamma \Phi}\left(\nabla^{\mu} \Phi\right)\left(\nabla_{\nu} \Phi\right)-\frac{\Omega}{2 \gamma \Phi} F^{\mu \lambda}\left(\nabla_{\nu} A_{\lambda}\right) .
\end{aligned}
$$

As mentioned, the ghost operator is not manifestly $\mathrm{U}(1)$ gauge-covariant though ln $\operatorname{det} \hat{M}$ certainly is. 
Now we follow the usual way of calculating the ln det. (Clearly, no doubling trick is needed, because ghost operator is simply given by (24).) Define:

$$
\hat{M}_{B}^{A}=\hat{K}_{B}^{A} \Delta+\hat{L}^{\lambda} \nabla_{\lambda}+\hat{P}_{B}^{A}
$$

so that

$$
\begin{gathered}
\left(\hat{K}^{-1}\right)_{B}^{A}=\left(\begin{array}{cc}
\frac{\Omega}{f} & \frac{\Omega-f}{f} A_{\nu} \\
0 & \delta_{\nu}^{\mu}
\end{array}\right), \quad \operatorname{det} \hat{K}=\frac{f}{\Omega}, \\
\operatorname{Tr} \hat{1}=1+\delta_{\mu}^{\mu}=3,
\end{gathered}
$$

and the explicit form of $\hat{L}^{\lambda}$ and $\hat{P}$ follows from (25). We introduce the matrices

$$
\hat{E}=\frac{1}{2} \hat{K}^{-1} \hat{L}^{\lambda}, \hat{\Pi}=\hat{K}^{-1} \hat{P}
$$

and make use of Eq. (20) to get

$$
\begin{aligned}
\Gamma_{g h, d i v}= & -i \operatorname{Tr} \ln \hat{M}_{g h}=-\frac{1}{2 \epsilon} \int d^{2} x \sqrt{g}\left\{3 R-\frac{\Omega}{2 \gamma \Phi} F^{2}+\left[\frac{C}{\gamma \Phi}-\frac{2}{\Phi}-\frac{\Omega^{\prime}}{\Omega}\right](\Delta \Phi)\right. \\
& \left.+\left[\frac{\Omega^{\prime 2}}{2 \Omega^{2}}-\frac{\Omega^{\prime \prime}}{\Omega}-\frac{\Theta}{\gamma \Phi}+\frac{2}{\Phi^{2}}+\frac{C^{2}}{4 \gamma^{2} \Phi^{2}}+\frac{C}{\gamma \Phi^{2}}\right]\left(\nabla_{\lambda} \Phi\right)\left(\nabla^{\lambda} \Phi\right)\right\} .
\end{aligned}
$$

The total divergent part of the effective action in the De Witt gauge is given by the sum of (23) and (28):

$$
\begin{aligned}
\Gamma_{d i v}=\quad & -\frac{1}{2 \epsilon} \int d^{2} x \sqrt{g}\left\{5 R-\frac{1}{\gamma \Phi} V+\frac{2}{C} V^{\prime}+\left[\frac{f^{\prime}}{2 C}+\frac{f}{4 \gamma \Phi}\right] F^{2}\right. \\
+ & {\left[\frac{f^{\prime}}{f}-2 \frac{\Omega^{\prime}}{\Omega}+\frac{C}{\gamma \Phi}-\frac{1}{C}-\frac{3}{\Phi}\right](\Delta \Phi) } \\
+ & {\left.\left[2 \frac{\Omega^{\prime 2}}{\Omega^{2}}-2 \frac{\Omega^{\prime \prime}}{\Omega}-\frac{f^{\prime 2}}{f^{2}}+\frac{f^{\prime \prime}}{f}+\frac{3}{\Phi^{2}}\right]\left(\nabla^{\lambda} \Phi\right)\left(\nabla_{\lambda} \Phi\right)\right\} . }
\end{aligned}
$$

This expression constitutes the main result of the present section.

A few remarks are in order. First of all, after dropping the surface divergent terms (which are kept in (29)) and putting $F^{2}=0$, the result (29) agrees with the calculations done in Ref. [15], and for $\xi=0(\gamma=-C / 2)$ with the result of Refs. [13,14] in the same gauge. The divergences of the Maxwell sector coincide with the results of Ref. [16], and for $\xi=0$ with those of Ref. [11]. As we can see, the Maxwell sector is $\Omega$-independent. (In fact, the Maxwell sector looks the same in the three different covariant gauges, as it will be discussed below.) 
Moreover, $\Gamma_{\text {div }}$ does not depend on $\Theta$, in accordance with the general results of Ref. [14] (notice that $\Theta$ is a particular case of the function $X(\Phi)$ introduced in Ref. [14]).

Before discussing renormalization, let us perform the calculation of $\Gamma_{d i v}$ in a different covariant gauge, which we here call $\Omega$-degenerate De Witt gauge. It is chosen as

$$
\chi^{*}=-\nabla^{\mu} Q_{\mu}
$$

in the Maxwell sector and the covariant De Witt gauge in the pure gravitational sector [15]:

$$
\chi^{\mu}=-\frac{C}{2 \gamma \Phi} \nabla^{\mu} \varphi+\frac{\Theta}{2 \gamma \Phi}\left(\nabla^{\mu} \Phi\right) \varphi+\frac{C}{4 \gamma \Phi}\left(\nabla^{\mu} \Phi\right) h-\nabla^{\nu} \bar{h}_{\mu \nu}-\frac{1}{\Phi}\left(\nabla^{\nu} \Phi\right) \bar{h}_{\nu}^{\mu} .
$$

The matrix $c_{A B}$ is chosen to be

$$
c_{A B}=\sqrt{g}\left(\begin{array}{cc}
f & 0 \\
0 & 2 \gamma \Phi g_{\mu \nu}
\end{array}\right) .
$$

The calculation can be done in direct analogy with the above case. The gravitationalMaxwell contribution to $\Gamma_{d i v}$ is given by (23) with all $\Omega$-terms dropped. The ghost operator is again non-diagonal:

$$
\begin{aligned}
& \hat{M}_{*}^{*}=\Delta, \quad \hat{M}_{*}^{\mu}=0 \\
& \hat{M}_{\nu}^{*}=A_{\nu} \Delta+\left(\nabla_{\lambda} A_{\nu}\right) \nabla^{\lambda}+\left(\nabla_{\nu} A_{\lambda}\right) \nabla^{\lambda}+\left(\nabla^{\lambda} \nabla_{\nu} A_{\lambda}\right), \\
& \hat{M}_{\nu}^{\mu}=\delta_{\nu}^{\mu} \Delta+\frac{1}{\Phi}\left(\nabla^{\lambda} \Phi\right) \delta_{\nu}^{\mu} \nabla_{\lambda}+\left(\frac{C}{2 \gamma \Phi}+\frac{1}{\Phi}\right) \nabla^{\mu} \\
& \quad-\left(\frac{C}{2 \gamma \Phi}+\frac{1}{\Phi}\right)+\frac{R}{2} \delta_{\nu}^{\mu}+\frac{C}{2 \gamma \Phi}\left(\nabla^{\mu} \nabla_{\nu} \Phi\right)-\frac{\Theta}{2 \gamma \Phi}\left(\nabla^{\mu} \Phi\right)\left(\nabla_{\nu} \Phi\right) .
\end{aligned}
$$

Using (33) we can find the ghost contribution in the form

$$
\begin{aligned}
& \Gamma_{g h, d i v}=-\frac{1}{2 \epsilon} \int d^{2} x \sqrt{g}\left\{3 R+\left[\frac{C}{\gamma \Phi}-\frac{2}{\Phi}\right](\Delta \Phi)\right. \\
&\left.+\left[\frac{C^{2}}{4 \gamma^{2} \Phi^{2}}+\frac{C}{\gamma \Phi^{2}}+\frac{2}{\Phi^{2}}-\frac{\Theta}{\gamma \Phi}\right]\left(\nabla^{\lambda} \Phi\right)\left(\nabla_{\lambda} \Phi\right)\right\} .
\end{aligned}
$$

Summing up, we find that the divergent part of the effective action in the $\Omega$-degenerate De Witt gauge is given by Eq. (29) discarding all $\Omega$-dependent terms.(It does not mean that $\Omega=0$.Rather,one sets $\Omega=$ const and after that setting it equal to zero .Otherwise,configuration space metric diverges.) This justifies the nickname " $\Omega$-degenerate" De Witt gauge given to the gauge (30)-(31). 
For completeness, we shall now write $\Gamma_{d i v}$ in the simplest covariant gauge $[11,16]$ :

$$
\chi^{*}=-\nabla^{\mu} Q_{\mu}, \quad \chi^{\mu}=-\frac{C}{2 \gamma \Phi} \nabla^{\mu} \varphi-\nabla^{\nu} \bar{h}_{\mu \nu},
$$

with the same $c_{A B}$ (32). An explicit evaluation in this case yields [16]:

$$
\begin{aligned}
\Gamma_{d i v}=- & \frac{1}{2 \epsilon} \int d^{2} x \sqrt{g}\left\{5 R-\frac{1}{\gamma \Phi} V+\frac{2}{C} V^{\prime}+\left[\frac{f}{4 \gamma \Phi}+\frac{f^{\prime}}{2 C}\right] F_{\mu \nu}^{2}\right. \\
& \left.+\left[\frac{f^{\prime}}{f}+\frac{1}{\Phi}-\frac{1}{C}\right] \Delta \Phi+\left[\frac{f^{\prime \prime}}{f}-\frac{f^{\prime 2}}{f^{2}}-\frac{1}{\Phi^{2}}+\frac{C}{\gamma \Phi^{2}}\right]\left(\nabla^{\lambda} \Phi\right)\left(\nabla_{\lambda} \Phi\right)\right\} .
\end{aligned}
$$

Thus we have calculated the one-loop divergences of the convenient effective action in three different covariant gauges: (i) the De Witt gauge, (ii) the $\Omega$-degenerate De Witt gauge, and (iii) the simplest covariant gauge. We see that $\Gamma_{d i v}$ is explicitly gauge dependent. Moreover, when the term (4) is present, $\Gamma_{d i v}$ depends on the parameter $\gamma$.

Let us now discuss the renormalizability of the theory off-shell. By dropping the surface terms in $\Gamma_{d i v}$ we see that all three gauges under consideration lead to the same off-shell effective action:

$$
\Gamma_{d i v}=-\frac{1}{2 \epsilon} \int d^{2} x \sqrt{g}\left\{\frac{2}{C} V^{\prime}-\frac{V}{\gamma \Phi}+\left[\frac{f^{\prime}}{2 C}+\frac{f}{4 \gamma \Phi}\right] F_{\mu \nu}^{2}+\frac{C}{\gamma \Phi^{2}}\left(\nabla^{\lambda} \Phi\right)\left(\nabla_{\lambda} \Phi\right)\right\} .
$$

Adding to the classical action (1) the corresponding counterterms ((37) with the opposite sign) we obtained the renormalized action. Choosing the one-loop renormalization of $g_{\mu \nu}$ as

$$
g_{\mu \nu}=\exp \left(-\frac{1}{2 \epsilon \gamma \Phi}\right) \widetilde{g}_{\mu \nu},
$$

we get the one-loop renormalized action in the following form

$$
S_{R}=-\int d^{2} x \sqrt{\widetilde{g}}\left[\frac{1}{2} \widetilde{g}^{\mu \nu} \partial_{\mu} \Phi \partial_{\nu} \Phi+C \widetilde{R} \Phi+V-\frac{V^{\prime}}{\epsilon C}+\frac{1}{4}\left(1-\frac{f^{\prime}(\Phi)}{4 \epsilon C}\right) \widetilde{g}^{\mu \alpha} \widetilde{g}^{\nu \beta} F_{\mu \nu} F_{\alpha \beta}\right] .
$$

The dilaton and the coupling $C$ do not get renormalized in the one-loop approximation.

It follows from (39) that the theory under discussion is one-loop multiplicatively renormalizable for the families of potentials:

$$
\begin{aligned}
V(\Phi)=e^{\alpha \Phi}+\Lambda, & f(\Phi)=e^{\beta \Phi}+f_{1}, \\
V(\Phi)=A_{1} \sin \Phi+B_{1} \cos \Phi & f(\Phi)=A_{2} \sin \Phi+B_{2} \cos \Phi
\end{aligned}
$$

where $\alpha, \Lambda, \beta, f_{1}, A_{1}, B_{1}, A_{2}$ and $B_{2}$ are arbitrary couplings. Let us recall that the black hole solutions for the Liouville like potentials of (40) have been studied in [4-7] in the case 
of dilaton gravity with $N$ scalars, and in $[9,11]$ in the case of 2 d dilaton-Maxwell gravity. Notice also the fact that, in contradistinction with 4d Einstein-Maxwell theory — which is not renormalizable [17] — in the theory under discussion we get off-shell renormalizability.

As we can see from the above calculations, in the three different gauges considered $\Gamma_{d i v}$ is given by three different expressions: the convenient effective action is gauge dependent. However, all the differences are contanined in the surface counterterms only. If we drop surface terms we surprisingly find that the effective action is the same in the three gauges considered. Hence, due to some reason - and at least in the gauges under discussion here - the gauge dependent divergences of the one-loop effective action in $2 \mathrm{~d}$ dilatonMaxwell gravity are included in the surface divergences.

Let us now study the on-shell limit of the effective action. Starting from (29), integrating by parts, keeping all the surface terms, and using the second and the third of the classical field equations (2), we get the on-shell divergences of the effective action:

$$
\Gamma_{\text {div }}^{o n-s h e l l}=-\frac{1}{2 \epsilon} \int d^{2} x \sqrt{g}\left\{3 R+\Delta\left[\ln \left(\frac{f}{\Phi^{3}}\right)-\ln \Omega^{2}+\frac{1}{C} \Phi\right]\right\} .
$$

Hence, one can see that the one-loop divergences of the on-shell effective action are just given by surface terms. In other words, the one-loop $S$-matrix is finite as in pure dilaton gravity. For comparison, remember that in 4d Einstein-Maxwell theory the one-loop $S$ matrix is not finite [17], while it is so in 4d Einstein theory [18]. The other interesting point to be noticed concerns the arbitrary gauge function $\Omega(\Phi)$. The fact that this function is present in (41) explicitly shows that on-shell surface divergences are gauge dependent. Indeed, had we started from $\Gamma_{d i v}$ in the $\Omega$-degenerate De Witt gauge, we would have got on-shell the same expression (41) without $\Omega$ terms. Using $\Gamma_{d i v}(36)$ on shell again leads to an expression different from (41) because of some surface divergences.

\section{One-loop unique effective action divergences in $2 \mathrm{~d}$ dilaton-Maxwell gravity}

In this section we will study the one-loop unique effective action for 2 d dilaton-Maxwell gravity. As is well known, this action is gauge invariant, gauge fixing and reparametrization independent. Actually, there is a whole family of unique effective actions [19-21]. However, all members of the family coincide in the one-loop approximation to quantum gravity, so there is no need to discuss here these differences in the definition of the action 
(review articles on the unique effective action are listed in Ref. [21]). Notice, however, that the unique effective action is configuration space metric dependent [23]. This is why, actually, the unique effective action does not solve the gauge dependence problem of the convenient effective action (as it had been claimed in the first works $[19,20]$ ), and the nickname "unique" does not have a proper sense. In fact we have a gauge dependence of the convenient effective action versus a configuration space metric dependence of the unique effective action. The unique effective action is still a useful covariant formalism which can add some information to the convenient effective action formalism. Moreover, there is still a hope to construct a physical off-shell effective action which would be really unique, along the direction started in [19,20]. Hence, the discussion of the unique effective action in situations where it can be compared with the convenient effective action is very useful. The finite parts of the unique effective action in $2 \mathrm{~d}$ gravity have been considered in Refs. [22]. The one-loop divergences in pure dilaton gravity have been obtained in Ref. [15]. In what follows, we will generalize the calculations of Ref. [15] to the case of dilaton-Maxwell gravity.

According to Refs. [19-21], one should add to the total quadratic expansion of the convenient effective action (19) in De Witt's gauge the correction

$$
-\frac{1}{2} \Gamma_{j k}^{i} \varphi^{j} \varphi^{k} \frac{\delta S}{\delta \varphi^{i}},
$$

where $\Gamma_{j k}^{i}=\left\{\begin{array}{c}i \\ j k\end{array}\right\}+T_{j k}^{i}[19-21]$ is the connection on the space of fields. This procedure will give the one-loop unique effective action. (Notice that as a consequence of the classical equations of motion the correction (42) is zero on-shell.)

Now we proceed with the calculation of the corrections introduced by (42). Notice that it is more convenient to use in what follows the dynamical variable $h_{\mu \nu}$, and not $\bar{h}_{\mu \nu}$ and $h$. The index $i$ in (42) runs through $\left\{Q_{\mu}, \varphi, h_{\mu \nu}\right\}$. For the configuration space metric (6), the Christoffel symbols $\left\{\begin{array}{c}i \\ j k\end{array}\right\}$ can be easily calculated. Part of them are listed in Ref. [15] (Eq. (26)); those do not change. We will write below the remaining non-zero components which appear in dilaton-Maxwell gravity:

$$
\begin{aligned}
& \left\{\begin{array}{c}
Q_{\alpha} \\
\varphi Q_{\mu}
\end{array}\right\}=\frac{\Omega^{\prime}}{2 \Omega} \delta_{\alpha}^{\mu}, \quad\left\{\begin{array}{c}
Q_{\alpha} \\
Q_{\mu} h_{\rho \sigma}
\end{array}\right\}=-\frac{1}{2} g_{\lambda \alpha} P^{\lambda \mu, \rho \sigma}, \\
& \left\{\begin{array}{c}
h_{\mu \nu} \\
Q_{\alpha} Q_{\beta}
\end{array}\right\}=\frac{\Omega}{2 \gamma \Phi} P_{\mu \nu}^{\alpha \beta}-\frac{\Omega^{\prime}}{2 C} g_{\mu \nu} g^{\alpha \beta} .
\end{aligned}
$$


Now, as has been discussed in [15] the local correction is given by

$$
\Gamma_{l o c}^{V D}=-\frac{1}{2 \epsilon} \int d^{2} x \sqrt{g}\left[\left(\hat{K}^{-1}\right)^{i j}\left\{\begin{array}{c}
k \\
i j
\end{array}\right\} S_{, k}\right],
$$

where $\hat{K}^{-1}$ is the same as before (see Eq. (17) but in new variables. In our case we easily get

$$
\begin{array}{rl}
\Gamma_{l o c}^{V D}=-\frac{1}{2 \epsilon} \int d^{2} & x \sqrt{g}\left\{R+\frac{1}{C} V^{\prime}+\left[\frac{1}{\gamma \Phi}+\frac{1}{C \Phi}-\frac{2 \Theta}{C^{2}}+\frac{\Omega^{\prime}}{C f}\right] V\right. \\
+ & {\left[\frac{2 \Theta}{C}-\frac{1}{C}-\frac{1}{\Phi}-\frac{C}{\gamma \Phi}-\frac{\Omega^{\prime}}{f}\right](\Delta \Phi)} \\
+ & \left.\left[\frac{\Theta}{2 C^{2}}-\frac{1}{4 \gamma \Phi}-\frac{1}{4 C \Phi}+\frac{f^{\prime}-\Omega^{\prime}}{4 C f}\right] f F^{2}\right\} .
\end{array}
$$

Now let us proceed with the evaluation of the non-local Vilkovisky-De Witt correction connected with the torsion $T_{j k}^{i}$ in (42). The generalized Schwinger-De Witt technique (see [26] for details) is very useful in this case. The application of this technique is based on having a De Witt covariant gauge, what we actually do (Sect. 2). As it has been discussed in detail in Ref. [15], the torsion (non-local) Vilkovisky-De Witt correction to the effective action in De Witt's gauge is given by

$$
\Gamma_{\tau, d i v}=-\left.\frac{i}{2} \operatorname{Tr} \hat{U}_{1}\right|_{d i v}
$$

where

$$
\begin{aligned}
& \hat{U}_{1 B}^{A}=\hat{N}^{A A^{\prime}} \nabla_{A^{\prime}}^{i}\left(\mathcal{D}_{i} \nabla_{A^{\prime \prime}}^{j}\right) S,{ }_{j} \hat{N}^{A^{\prime} B^{\prime}} c_{B^{\prime} B}, \quad \hat{N}^{A B}=\left(\hat{N}_{A B}\right)^{-1} \\
& \hat{N}_{A B}=-C_{A A^{\prime}} \hat{M}_{g h B}^{A^{\prime}}=-\Omega \sqrt{g}\left(\begin{array}{cc}
1 & A_{\mu} \\
A_{\nu} & A_{\mu} A_{\nu}+2 \gamma \Phi / \Omega
\end{array}\right) \Delta+\cdots
\end{aligned}
$$

where $\hat{M}_{g h}$ and $c_{A B}$ are given by (25) and (9), respectively, $\nabla_{B}^{j}$ are the gauge generators, $\mathcal{D}_{i}$ the covariant derivative in the space of fields $\left(\mathcal{D}_{i}\right.$ is constructed with the Christoffel symbols), and the lower derivative terms in the third expression (47) may be discarded.

Introduce the notations

$$
\begin{aligned}
& c_{A(x) B(y)} \equiv c_{A B}(x) \delta(x-y), \\
& \nabla_{A(y)}^{i(x)}=t_{A}^{i} \nabla_{x} \delta(x-y)+\ldots, \\
& \hat{N}^{A(x) B(y)}=\mathcal{N}^{A B}(x) \frac{1}{\Delta_{x}} \delta(x-y)+\ldots, \\
& \mathcal{D}_{i(x)} \nabla_{A(z)}^{j(y)}=\mathcal{D}_{i A}^{j}(y) \delta(y-z) \nabla_{y} \delta(y-x)+\cdots,
\end{aligned}
$$


where the lower order derivatives may again be omitted. Then, the divergent structure of $\hat{U}_{1}$ becomes evident (see Ref. [15])

$$
\begin{aligned}
& \hat{U}_{1 B(y)}^{A(x)}=\mathcal{U}_{B}^{A}(x) \nabla_{x} \nabla_{x} \frac{1}{\Delta_{x}^{2}} \delta(x-y)+\ldots, \\
& \mathcal{U}_{B}^{A}=-\mathcal{N}^{A A^{\prime}} t_{A^{\prime}}^{i} \mathcal{D}_{i A^{\prime \prime}}^{j}{ }_{, j} \mathcal{N}^{A^{\prime \prime} B^{\prime}} c_{B^{\prime} B},
\end{aligned}
$$

and $[26]$

$$
\left.\nabla_{\mu(x)} \nabla_{\nu(x)} \frac{1}{\Delta_{x}^{2}} \delta(x-y)\right|_{y \rightarrow x} \longrightarrow-\frac{i}{2 \epsilon} \sqrt{g(x)} g_{\mu \nu}(x) .
$$

The functional trace in (46)

$$
\operatorname{Tr} \ldots \equiv \int d^{2} x \sum_{A} \lim _{y \rightarrow x} \ldots
$$

can be performed to yield

$$
\begin{aligned}
& \mathcal{U}_{A}^{A}=-R^{A B} t_{B}^{i} \mathcal{D}_{i A^{j}} S,_{i}, \\
& R^{A B}=\mathcal{N}^{A A^{\prime}}{ }_{A_{A^{\prime} B^{\prime}}} \mathcal{N}^{B^{\prime} B}=\frac{1}{2 \gamma \Phi \sqrt{g}}\left(\begin{array}{cc}
\frac{2 \gamma \Phi}{f}+A^{\lambda} A_{\lambda} & -A^{\nu} \\
-A^{\mu} & g^{\mu \nu}
\end{array}\right) .
\end{aligned}
$$

Calculating the covariant derivatives and using (46) and (49)-(52), we obtain

$$
\begin{aligned}
& \Gamma_{\tau, d i v}=- \frac{1}{2 \epsilon} \int d^{2} x \sqrt{g}\left\{\frac{1}{C \sqrt{g}} \frac{\delta S}{\delta \Phi}+\left(\frac{\Omega^{\prime}}{2 C f}+\frac{1}{C \Phi}-\frac{\Theta}{C^{2}}\right) \frac{1}{\sqrt{g}} g_{\mu \nu} \frac{\delta S}{\delta g_{\mu \nu}}\right\} \\
&=-\frac{1}{2 \epsilon} \int d^{2} x \sqrt{g}\left\{-R-\frac{1}{C} V^{\prime}+\left[\frac{\Theta}{C^{2}}-\frac{1}{C \Phi}-\frac{\Omega^{\prime}}{2 C f}\right] V\right. \\
&\left.+\left[\frac{\Omega^{\prime}-2 f^{\prime}}{8 C}+\frac{f}{4 C \Phi}-\frac{f \Theta}{4 C^{2}}\right] F^{2}+\left[\frac{\Omega^{\prime}}{2 f}+\frac{1}{C}+\frac{1}{\Phi}-\frac{\Theta}{C}\right](\Delta \Phi)\right\} .
\end{aligned}
$$

The total Vilkovisky-De Witt correction to the divergent part of the effective action (29) is given by the sum of (45) and (53). Notice that this sum vanishes on shell and that, discarding the Maxwell sector, it coincides with the result obtained in Ref. [15].

Finally, the one-loop unique effective action is given by the sum of (29), (45), and (53):

$$
\begin{aligned}
\Gamma_{\text {unique }, \text { div }}= & -\frac{1}{2 \epsilon} \int d^{2} x \sqrt{g}\left\{5 R+\frac{2}{C} V^{\prime}+\left[\frac{\Omega^{\prime}}{2 C f}-\frac{\Theta}{C^{2}}\right] V\right. \\
+ & {\left[\frac{f^{\prime}}{2 C}-\frac{\Omega^{\prime}}{8 C}+\frac{f \Theta}{4 C^{2}}\right] F^{2} }
\end{aligned}
$$




$$
\begin{aligned}
& +\left[\frac{f^{\prime}}{f}-2 \frac{\Omega^{\prime}}{\Omega}-\frac{\Omega^{\prime}}{2 f}+\frac{\Theta}{C}-\frac{1}{C}-\frac{3}{\Phi}\right](\Delta \Phi) \\
& \left.+\left[2 \frac{\Omega^{\prime 2}}{\Omega^{2}}-2 \frac{\Omega^{\prime \prime}}{\Omega}-\frac{f^{\prime 2}}{f^{2}}+\frac{f^{\prime \prime}}{f}+\frac{3}{\Phi^{2}}\right]\left(\nabla^{\lambda} \Phi\right)\left(\nabla_{\lambda} \Phi\right)\right\} .
\end{aligned}
$$

It is evident that the unique effective action (54) on-shell leads to the same expression (41) as the convenient effective action (29). The configuration space metric dependence (through the arbitrary functions $\Theta(\Phi)$ and $\Omega(\Phi)$ is seen explicitly in (54).

What about the renormalization of the unique effective action off-shell? Choosing the renormalization of $g_{\mu \nu}$ as

$$
g_{\mu \nu}=\exp \left[\frac{\Omega^{\prime}}{4 C \epsilon f}-\frac{\Theta}{2 C^{2} \epsilon}\right] \widetilde{g}_{\mu \nu},
$$

we get the renormalized effective action in the form

$$
\begin{aligned}
S_{R} & =-\int d^{2} x \sqrt{\widetilde{g}}\left[\frac{1}{2} \widetilde{g}^{\mu \nu} \partial_{\mu} \Phi \partial_{\nu} \Phi+C \widetilde{R} \Phi+V(\Phi)-\frac{V^{\prime}}{\epsilon C}\right. \\
& \left.+\frac{1}{4}\left(1-\frac{f^{\prime}(\Phi)}{\epsilon C f(\Phi)}\right) \widetilde{g}^{\mu \alpha} \widetilde{g}^{\nu \beta} F_{\mu \nu} F_{\alpha \beta}\right] .
\end{aligned}
$$

Thus, the one-loop renormalization of the metric tensor (55) in this case is different from the one corresponding to the convenient effective action formalism. However, the renormalized effective action (56) looks exactly the same, and leads to the same class of renormalizable dilaton and Maxwell potentials as given in (40).

\section{Conclusions}

Summing up, we have discussed the one-loop renormalization structure of $2 \mathrm{~d}$ dilatonMaxwell gravity. The one-loop convenient effective action has been found in three different covariant gauges. However, the gauge dependence appears only in surface divergent terms. Moreover, the on-shell effective action is given by surface divergent terms only (on-shell finiteness); however, these terms are still gauge dependent. The one-loop divergences of the unique effective action — which is known to be gauge fixing independent and parametrization independent - have been found. This effective action is explicitly demonstrated to be configuration space metric dependent.

The off-shell renormalizability leads to the same renormalizable dilaton and Maxwell potentials both for the case of the convenient and for the unique effective action. It is 
very interesting to notice that the heterotic string effective action is described as just a particular case of the renormalizable dilaton-Maxwell theory (1). It would be important to examine $2 \mathrm{~d}$ quantum cosmology [30] for this renormalizable model.

The other topic which deserves further study has to do with the generalization of the model to include other dilaton-matter systems. As we show in the Appendix for the case of the $2 \mathrm{~d}$ dilaton-Yang-Mills theory, there appears only a trivial (surface divergence) correction to the one-loop effective action, as compared with the dilaton-Maxwell theory. Hence, there are no loop corrections from the gravitational coupling $C$ to Yang-Mills coupling (which is dimensional). However, if we add fermions to the theory, we can expect such corrections to appear in the four-fermion coupling [28] and in the Pauli coupling [29]. Both these coupling constants are dimensionless and this would not be in contradistinction with renormalizability. Moreover, the chiral anomaly structure can be very interesting in this case. Work along this line is in progress.

\section{Acknowledgments}

We would like to thank I. Antoniadis, F. Englert, R. Kantowski, C. Marzban, F. Mazzitelli, N. Mohammedi, A.A. Slavnov, and I.V. Tyutin for useful discussions at different stages of this work. S.D.O. wishes to thank the Japan Society for the Promotion of Science (JSPS, Japan) for financial support and T. Muta and the Particle Physics Group at Hiroshima University for kind hospitality. E.E. has been supported by DGICYT (Spain), research project PB90-0022, and by the Alexander von Humboldt Foundation (Germany).

\section{A Appendix}

In this Appendix we extend the calculations of Sect. 2 to the case of dilaton-Yang-Mills gravity. We start again from action (1) with the only change of $F_{\mu \nu}$ by $F_{\mu \nu}^{a}$, where

$$
F_{\mu \nu}^{a}=\nabla_{\mu} A_{\nu}^{a}-\nabla_{\nu} A_{\mu}^{a}+f_{b c}^{a} A_{\mu}^{b} A_{\nu}^{c}
$$

being $f_{a b c}$ antisymmetric, and the gauge group is chosen to be simple and compact (for a recent discussion of $2 \mathrm{~d}$ quantum gauge theory see [27] and references therein).

The background field method will be employed, exactly as in Sect. 2 (only $Q_{\mu}$ must 
be substituted by $Q_{\mu}^{a}$ ) and the simplest covariant gauge is considered, where

$$
c_{A B}=\sqrt{g}\left(\begin{array}{cc}
f \gamma_{a b} & 0 \\
0 & 2 \gamma \Phi g_{\mu \nu}
\end{array}\right)
$$

and $\gamma_{a b}=-f_{a d}^{c} f_{b c}^{d}$ is the positive Killing metric. The calculation can be repeated along the same lines as in Sect. 2 (see also Ref. [16]). Actually, only the $Q Q$-sector is going to change. In comparison with [16], the following components of $\hat{E}^{\lambda}$ and $\hat{\Pi}$ are different

$$
\begin{aligned}
& \left(\hat{E}^{\lambda}\right)_{1}^{1}=\frac{f^{\prime}}{2 f} \delta_{b}^{a}\left[g^{\alpha \lambda}\left(\nabla_{\rho} \Phi\right)-g_{\rho}^{\lambda}\left(\nabla^{\alpha} \Phi\right)+g_{\rho}^{\alpha}\left(\nabla^{\lambda} \Phi\right)\right], \\
& \hat{\Pi}_{1}^{1}=2 f_{c b}^{a} F_{\rho}^{c \alpha}-\delta_{b}^{a} R_{\rho}^{\alpha} .
\end{aligned}
$$

Finally, we obtain the following expression for the one-loop effective action

$$
\begin{aligned}
\Gamma_{d i v}=- & \frac{1}{2 \epsilon} \int d^{2} x \sqrt{g}\left\{R(N+4)+\frac{2}{C} V^{\prime}-\frac{1}{\gamma \Phi} V+\left(\frac{f^{\prime}}{2 C}+\frac{f}{4 \gamma \Phi}\right) F_{\mu \nu}^{a 2}\right. \\
& +N\left(\frac{f^{\prime}}{f}+\frac{1}{\Phi}-\frac{1}{N C}\right)(\Delta \Phi) \\
& \left.+N\left(\frac{f^{\prime \prime}}{f}-\frac{f^{\prime 2}}{f^{2}}-\frac{1}{\Phi^{2}}+\frac{C}{N \gamma \Phi^{2}}\right)\left(\nabla^{\lambda} \Phi\right)\left(\nabla_{\lambda} \Phi\right)\right\}
\end{aligned}
$$

where $N=\delta_{a}^{a}$, and in evaluating the ghost contribution we have taken into account that $\Gamma_{g h, d i v}$ is given by the corresponding expression from Ref. [16], with the only change of the $R$-term by $\frac{R}{3}(N+8)$ (in the Abelian case $N=1$ ).

In fact, we have shown that the as long as $Q_{\mu}^{a}$ does not mix with the other fields via gauge conditions, the non-Abelian case may be obtained from the Abelian one by adding quite simple corrections, $\delta \Gamma$,

$$
\delta \Gamma=-\frac{N-1}{2 \epsilon} \int d^{2} x \sqrt{g}\left\{R+\nabla_{\lambda}\left[\left(\frac{f^{\prime}}{f}+\frac{1}{\Phi}\right)(\Delta \Phi)\right]\right\} .
$$

A most remarkable point is that this correction is just a surface term.

In particular, for the $\Omega$-degenerate De Witt gauge, the one-loop effective action in $2 \mathrm{~d}$ dilaton-Yang-Mills gravity is given by the sum of (60) and of expression (29) with the $\Omega$-terms excluded. The calculation of the unique effective action in 2 d dilaton-Yang-Mills gravity is more complicated and will not be done here. 


\section{References}

[1] S.W. Hawking, Commun. Math. Phys. 43 (1975) 199.

[2] R.M. Wald, Commun. Math. Phys. 45 (1975) 9.

[3] P. Davis, S. Fulling, and W. Unruh, Phys. Rev. D13 (1976) 2720.

[4] C.G. Callan, S.B. Giddings, J.A. Harvey and A. Strominger, Phys. Rev. D45 (1992) 1005; S.B. Giddings and W.M. Nelson, Phys. Rev. D46 (1992) 2486.

[5] S.W. Hawking, Phys. Rev. Lett. 69 (1992) 406; L. Susskind and L. Thorlacius, Nucl. Phys. B382 (1992) 123; B. Birnir, S.B. Giddings, J.A. Harvey and A. Strominger, Phys. Rev. D46 (1992) 638; T. Banks, A. Dabholkar, M.R. Douglas and M. O'Loughlin, Phys. Rev. D45 (1992) 3607; K. Hamada, preprint UT-Komaba 927 (1992); J.G. Russo, L. Susskind and L. Thorlacius, Phys. Lett. B292 (1992) 13; Phys. Rev. D46 (1992) 3444; G.M. Gibbons and M.J. Perry, preprint hep-th 9204090 (1992); S.W. Hawking and J.M. Stewart, preprint DAMTP/R-92/37 (1992); R.B. Mann, Gen. Relat. Grav. 24 (1992) 433; T.Muta,S.D.Odintsov and H.Sato, Mod. Phys. Lett. A7 (1992) 3765; N. Sakai, preprint TIT-HEP-205 (1992).

[6] S.P. de Alwis, preprint hep-th 9207095 (1992); A. Bilal and C. Callan, preprint PUPT-1320 (1992); K. Hamada and A. Tsuchiya, preprint UT-Komaba 92-14 (1992); S.B. Giddings and A. Strominger, preprint hep-th 9207034 (1992); F. Belgiorno, A.S. Cattaneo, F. Fucito, and M. Martellini, preprint ROM2F-92-52 (1992).

[7] J.A. Harvey and A. Strominger, preprint EFI-92-41 (1992).

[8] E. Witten, Phys. Rev. D44 (1991) 314; G. Mandal, A. Sengupta and S. Wadia, Mod. Phys. Lett. A6 (1991) 1685; R. Dijkgraaf, H. Verlinde and E. Verlinde, Princeton preprint IASSNS-HEP 91/22 (1991).

[9] O. Lechtenfeld and C. Nappi, Phys. Lett. B288 (1992) 72; C. Nappi and A. Pasquinucci, Mod. Phys. Lett. A7 (1992) 3337; S. Nojiri and I. Oda, preprint hep-th 9207077 (1992).

[10] G.W. Gibbons, Nucl. Phys. B207 (1982) 337; G.W. Gibbons and K. Maeda, Nucl. Phys. B298 (1988) 74; A. Shapere, S. Trivedi and F. Wilczek, Princeton preprint IASSNS-HEP-91/33 (1991). 
[11] E. Elizalde and S.D. Odintsov, Mod. Phys. Lett. A8 (1993) 33; Nucl. Phys. B, to appear.

[12] S.D. Odintsov and I.L. Shapiro, Phys. Lett. B263 (1991) 183; Mod. Phys. Lett. A7 (1992) 437.

[13] F.D. Mazzitelli and N. Mohammedi, Nucl. Phys. B, to appear.

[14] S.D. Odintsov and I.L. Shapiro, Int. J. Mod. Phys. D, to appear.

[15] R. Kantowski and C. Marzban, Phys. Rev. D, to appear.

[16] E. Elizalde, S. Naftulin and S.D. Odintsov, preprint hep-th 9212139 (1992).

[17] S. Deser and P. van Nieuwenhuizen, Phys. Rev. D10 (1974) 401.

[18] G. 't Hooft and M. Veltman, Ann. Inst. H. Poincare 20 (1974) 69.

[19] G.A. Vilkovisky, Nucl. Phys. B234 (1984) 125.

[20] B.S. De Witt, in Quantum Field Theory and Quantum Statistics, eds. I.A. Batalin, C.J. Isham and G.A. Vilkovisky, Hilger, Bristol, 1987.

[21] R. Kobes, G. Kunstatter and D.J. Toms, in Proceedings of the John Hopkins Workshop on Current Problems in Particle Theory 12, eds. G. Domokos and S. KovesiDomokos, World Sci., Singapore, 1989; A. Rebhan, ibidem, 1989; I.L. Buchbinder and S.D. Odintsov, Fortschr. Phys. 37 (1989) 225; S.D. Odintsov, Fortschr. Phys. 38 (1990) 371;

G. Kunstatter, Class. Quant. Grav. 9 (1992) 157.

[22] E. Elizalde and S.D. Odintsov, Mod. Phys. Lett. A7 (1992) 2369; S.D. Odintsov and I.N. Schevchenko, Z. Phys. C56 (1992) 315.

[23] S.D. Odintsov, Phys. Lett. B262 (1991) 394.

[24] B.S. De Witt, Dynamical Theory of Groups and Fields, Gordon and Breach, NY, 1965.

[25] E. Elizalde and A. Romeo, Int. J. Mod. Phys. A5 (1990) 1653.

[26] A.O. Barvinsky and G.A. Vilkovisky, Phys. Rep. 119 (1985) 1. 
[27] E. Witten, preprint IASSNS-HEP-92/15 (1992).

[28] D.J. Gross and A. Neveu, Phys. Rev. D10 (1974) 2706.

[29] P. Bedaque, A. Das and W. Huang, preprint UR-1204 (1991).

[30] F.D. Mazzitelli and J.G. Russo, preprint UTTG-28-92 (1992); M. Yoshimura, preprint TU/92/146 (1992).

[31] R. Mann, A. Sheikh and L. Tarasov, Nucl. Phys. B341 (1990) 134; R. Mann, S. Morsink, A. Sikemma and T.G. Steele, Phys. Rev. D43 (1991) 3948. 